\title{
An Interactive E-Tool for Maintaining and Monitoring Medical Records using Blockchain Technology
}

\author{
S.Baghavathi Priya ${ }^{1}$, M.Muni Prasanna Kumar ${ }^{2}$, A.S.Reshma ${ }^{3}$, K.Sahana ${ }^{4}$ \\ \{baghavathipriya.s@ rajalakshmi.edu.in ${ }^{1}$, muniprasannakumar.m.2017.it@ rajalakshmi.edu.in² ${ }^{2}$, \\ reshma.as.2017.it@rajalakshmi.edu.in ${ }^{3}$, $\underline{\text { sahana.k.2017.it@ rajalakshmi.edu.in }}^{4}$ \}
}

Department of CSE, Rajalakshmi Engineering College ${ }^{1}$, Department of IT, Rajalakshmi Engineering College $2,3,4$

\begin{abstract}
Healthcare has become one of India's largest sectors. The growing concern is to find the right doctor and adhere to medication as prescribed. This brings the necessity for a medium where one could find doctors and book appointments for consultation. The proposed system provides a medium to discover doctors under certain specializations sorted based on user reviews, location, and availability and book appointments for consultation. The doctor will be able to fill in a form replacing the traditional paper prescriptions that can be accessed by the patient at any time. All the other medical reports can also be stored in this application which will be secured with a password known only to the patient. For editing a prescription given, two passwords: 1 - Patient, 1- Doctor will have to be used. In addition to password protection, storing the records through blockchain will ensure data integrity and provide more security. This system also provides a way to make patients get alerted for taking pills, for next reviews, tests to be taken, and surgery dates. These reminders can be very useful to elderly people to ensure medication adherence. This application also comes with some additional features like an emergency button, tapping which a call will be connected to the Doctor.
\end{abstract}

Keywords: Book appointment, Medical records, Reminders, Security, medication adherence, Blockchain.

\section{Introduction}

Health refers to the well-being of an individual both mentally and emotionally. Health is directly related to productivity in ones job leading to absenteeism or presentism. In order to maintain a good health comes the health care. The demand for health care is unlike the demand for most consumer products and services. Buying products or services comes from direct desire whereas healthcare is treated to be an option. This desire for health care is not derived directly from the consumption of the medical procedures themselves. But, it comes from the direct value of improved health that is produced by health care. With the fast-paced world, time spent on one's health is minimal. Medication non-adherence and non-compliance is a growing concern. This system is an attempt to address these issues and also help people find and manage their medical records easily. This system provides a way to search doctors based on their specializations specific to a location, book appointments. A secure system to store medical records with 2 factor authentication and also to set reminders.

The proposed system overcomes some of the major challenges in the existing applications especially this system concentrates on providing more security to the user data by providing 2 
factor authentication while the recent survey states that $71 \%$ of the applications are vulnerable security [21]. Integrating block chain technology in storing medical records will play a major role in maintaining data security and integrity. This will also ensure that the prescriptions aren't tampered by others. An interactive tool that is helpful for both patients and doctors in order to connect with each other and also help patients maintain their personal health records and improve medication adherence. To ensure security blockchain might be used. Blockchain is a way of storing sensitive data in a decentralized format. It uses SHA -256 algorithm to encrypt the data to be stored. SHA -256 algorithm returns a constant has of 256 bits irrespective of the input data size. This mitigation method would make it harder and even impossible for hackers to penetrate data storage systems. Many storage service companies are assessing ways in which blockchain can protect data from hackers.

\section{Related Work}

IoT-based Health Monitoring System with Medicine Reminder using Raspberry Pi is an health monitoring device. Pulse and temperature sensors are attached to monitor patient's pulse and temperature and quantify them. Based on the predefined values set by the user, at any point if the obtained value exceed the specified value, the system sends the predefined message to the doctor immediately via the IoT. It was developed by M. Amru et al [1], in 2020. The time, date and number of capsules are provided to the system by using the personal computer and the device is turned on after it calculates and reminds the timing of the capsule consumption. It also gives the heartbeats and temperature of the user. The reminders are made on time. As it creates a bell sound it is easy to identify the reminders. Stores the prescription as compressed image. The capsule timings are entered by the user. It is important to have a personal computer to enter the capsules. Other than pills reminders there is no searching of doctor applications. Cost of the hardware requirements are higher. Need to take care of hardware equipment.

A Smartphone Based Application for Health Care Management System is an Android application for booking an appointment, checking availability of beds, with pills remainder. This paper was proposed by Dr. Sangeetha. V et al [2], in the year 2020. Patients can find the right doctor based on ratings, location, and feedback from other patients. They can set reminders for medicines or drinking enough water. A person who is too busy or lazy to go to the clinic can chat with the respective doctor online at any time of the day. Anyone can upload prescriptions, medical reports etc. to keep them safe and hand. The patient can choose hospitals based on cost and quality. Application will provide an emergency button to make an emergency call for an ambulance. Provides facilities to choose the doctor based on the category of the disease and making an appointment with the doctor.Information about the hospitals and presenting their locations. - Application will provide a pills remainder. Major drawback is that prescriptions are uploaded as a picture which makes the patients difficult to understand and it also takes more space to save the images.

Fall Detection Application for the Elderly in the Family Heroes System proposed byMoustafaFayad et al [3]. This is a machine learning approach to recognize activities and obtain results based on the threshold of the movements using the wearable sensors. Approaches of deep learning are gaining popularity especially for visual sensors and sensor fusion and are becoming the state-of-the-art for fall detection and other activity recognition. For fall detection Deep reinforcement learning is another promising technique. The main 
objective is to monitor elderly people in home or instituitions. This fall detector can also detect if some one is in same position for longer than normal which could mean that there is some problem, the caretaker can be alerted immediately. This can help them to check on the elderly people. The major challenge user faces are the rarity of data of real falls, detection in real-time, security and privacy, platform of sensor fusion, limitation of location, scalability and flexibility.

Effective Online Medical Appointment System is a web application to view doctors and book appointments according to doctor's schedule that overcomes the issue of managing and booking appointments according to user's choice or demands. Here the user can select good doctors by viewing their details and reviews. S.Hema Kumar et al [4], developed this application in 2019. The doctor can see the patient request and sends the notification to the patients if the appointment is available. But, it does not provide a medium to store prescriptions and set reminders. Patient cannot choose the appointment time. This is the major drawback.

Android Application of Hospital for Karjat Taluka is an android application for Patients Appointment System and to view and manage appointments.This paper was published by Rahul Raj Verma et al [5].This system will provide the patient to schedule an appointment with the doctor. It also provides the patient a medium to interact and communicate with doctors without making any physical appointments with them. It is a medium to book and manage doctor appointments. Also provides chat options to connect with a doctor. Only the doctor can see the history of the patient.Itis made only for one specific hospital. There is no feature for searching other doctors or to set reminders.

\section{Existing Systems}

All the system available are mostly an android application. It doesn't have proper security for storing data. The users are either doctor and patient or admin and patient. Anyone can register as doctor and access the records. There will be no confirmation message from doctor after booking appointments.

Pills reminder is also not efficient since notifications will be sent to registered emails. More space needed since prescriptions should be scanned and uploaded. Incase of emergency, there is no provision to call doctor immediately. If the doctor is not available due to unavoidable conditions, patients will not be notified about the change in appointment timing. All the existing systems are centralized and specific to one hospital.

\section{Proposed System Architecture}

The main goal of the systems is to make it easier for the patients to find and book appointments with doctors based on the problems they're facing, from a single platform. The patient can view a list of doctors and filter doctors based on specializations and needs. The medical history of each and every patient will be maintained in a centralized store allowing for easy access. Data of the patients will be secured, meaning that the patient will not have to worry about the leakage of personal data. The doctor will be able to fill in and upload prescriptions which can be viewed by the patient through a two factor authentication. Reminders will be based on the timings set in the prescriptions to notify the patient 
accordingly. The medicals records are stored using encryption algorithm to ensure that the records cannot be tampered or viewed by third party.

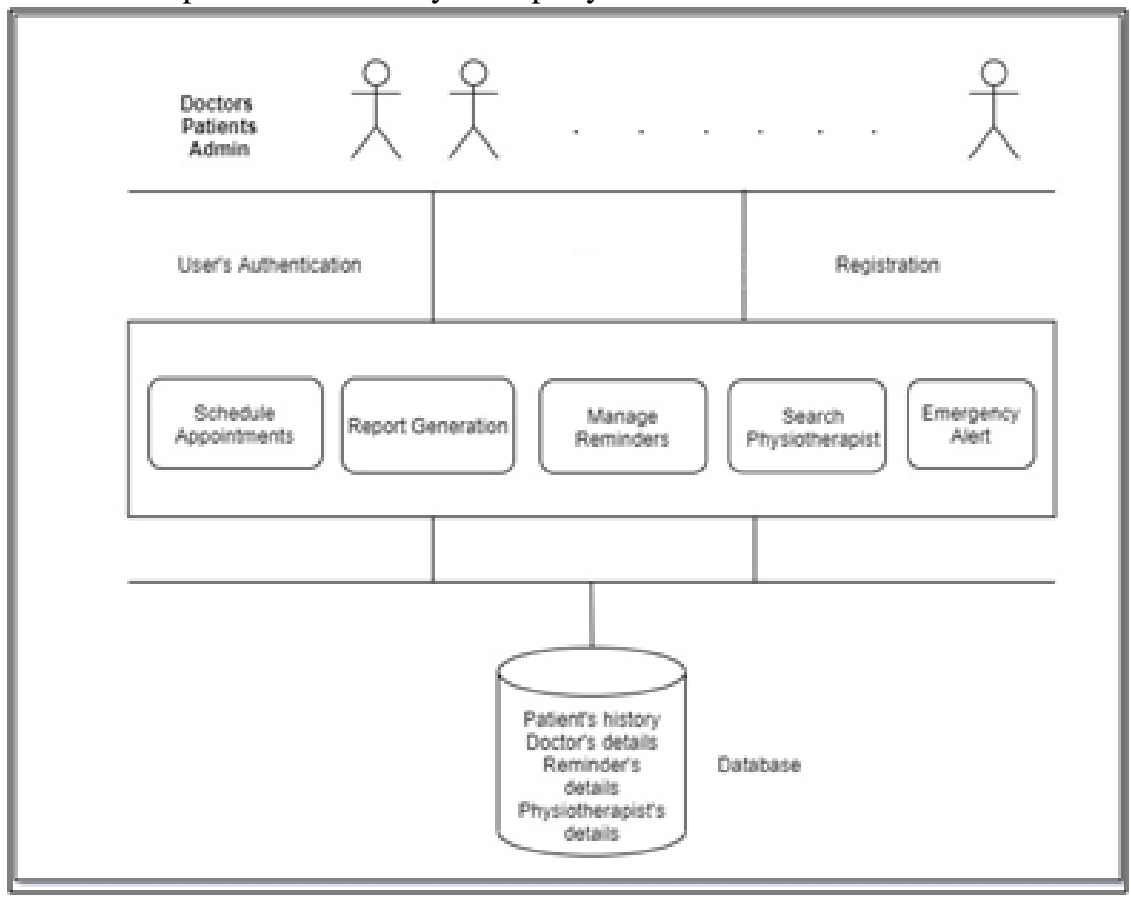

Figure 3.1 Proposed Architecture

The above diagram explains how this architecture works. User can identify themselves as Patient, Doctor and Admin and authenticate by entering login credentials. The role of the admin is to maintain patient and doctor lists. Doctor can upload prescriptions, manage the appointments and contact the patient whereas the patient can search doctors, book appointments and upload/View medical records and also make use of the reminders.

The main goal is to provide security to the records stored. This is done by encrypting the details using SHA - 256 encryption algorithm. Only the authorised person with correct credentials will be able to access them.

\section{Implementation}

The proposed system is to develop both web and android applications that provide a medium to make interaction between doctor and patient easier. Also, an automatic alarm ringing system that can be used to set reminders to take pills, take tests or to go for appointments. The system will also help to find all the prescriptions and reports in one place. Initially, users will be directed to a login page where a new user can register by entering their personal details. Every user will be given an unique user ID and using their password, one can login. There are three types of users, the administrator, the doctor and the patient. The administrator does the role of managing the doctors. If any doctor wants to get started with this platform, then he/she has to go through the admin, after which, if his/her credentials are approved, any patient can book an appointment with that doctor. 
An index of doctors is developed, so that the patients can search for doctors all over the country. This process has to start from a small area and then branch out to other areas, and eventually other cities. The registered doctors will have a time window setup from their dashboard, and a patient is allowed to book appointments only between the time windows that the doctor entered. The doctor can also update his status as unavailable, in such cases, the patients who've booked an appointment will be notified of the cancelled appointments.

On medical consultation, the doctor will update the prescription to the online form, which will set up reminders based on the inputs provided by the doctor, making sure the patient takes the medication correctly. This reminder system also works for reminding the patients of their upcoming tests, surgeries and the next reviews. For editing a patient's medical records, both the patient and the doctor have to authorize the edit. This prevents any unauthorized edits to the medical records. Either the doctor or patient can view the records, but if a new doctor wants access to the records, then the patient has to authorize the request. This provides Security on the Logical layer. To secure data on the data layer, all the patient data is hashed so that the data is not immediately legible. All the patient's data is tied to an ID, and no personal information is stored along with the medical data, which will prevent the exploitation of the data even in case of unauthorized access to the data.

The proposed system consists of five modules.

\section{Login Module:}

2. Appointment Module:

- Three different types of login: Admin, Doctor, Patient.

- Initially the user registers themselves as doctor or patient

- With the correct credentials, the user is logged into the system successfully.

- Respective dashboard is displayed.

- Patients will be able to view list of doctors based on specialisation and user reviews.

- For the selected doctor, based on availability appointments can be booked.

\section{Reminder Module:}

- The doctor will be given a form through which prescription can be filled.

- Based on the prescription given, reminders are set for pills, lab tests and next reviews.

- Patient will get alerted for the reminders set.

4. Authentication - Blockchain:

- Doctors will be given a form with which they can upload the prescription.

- Patient and doctors can access the records using a One time Verification Code or a Password they set initially.

\section{Integration of web interface:}

- Web application provides some of the features that the android application provides such as searching doctors and uploading/viewing medical records.

- The doctor will be given a prescription form where one can enter all the details about tablets - timings, dosage etc.and also enter the details of the lab tests that need to be taken. 


\section{Result And Discussions}

- This work mainly focused on providing security to the patient records, provide a medium to store, and access the same easily.

- The data is encrypted using SHA - 256 algorithm before storing it into the database.

- In comparison with the existing system, the proposed system provides an user friendly interface - both web and mobile.

- In addition to the features provided by the existing system like appointment booking and reminders, the proposed system ensures integrity of the message being stored.

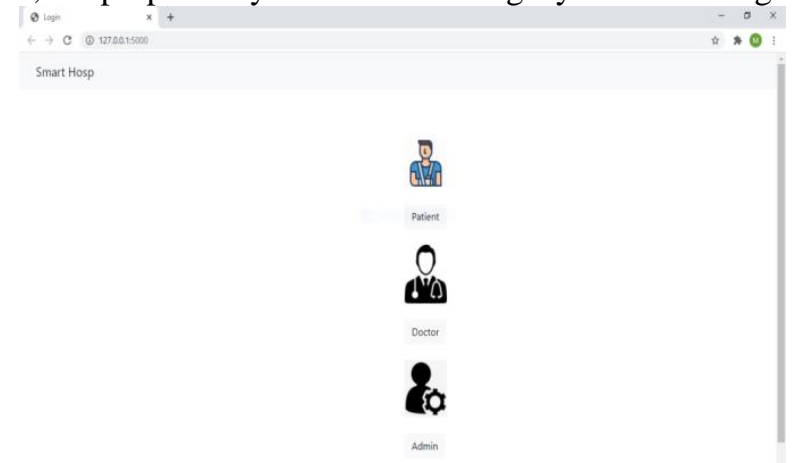

Fig 5.1 Web Login

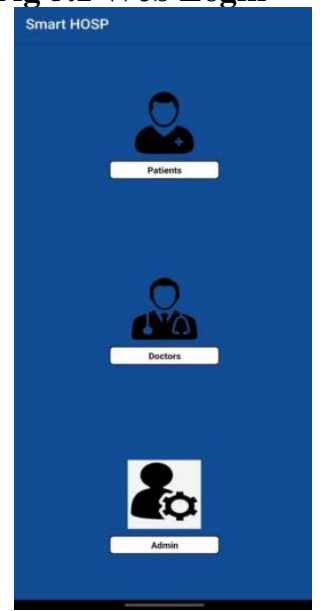

Fig 5.2 Mobile login 


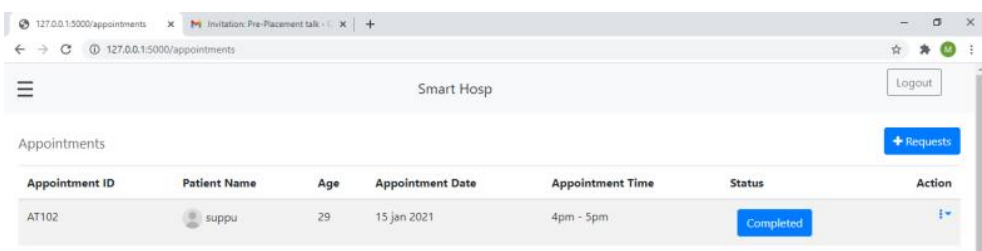

Fig 5.3 Doctor appointment dashboard

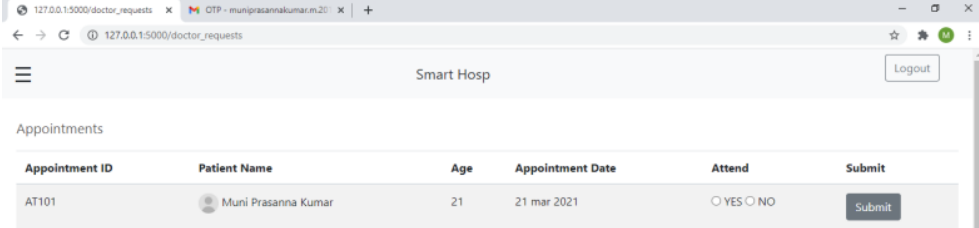

Fig 5.4 Appointment confirmation by Doctor

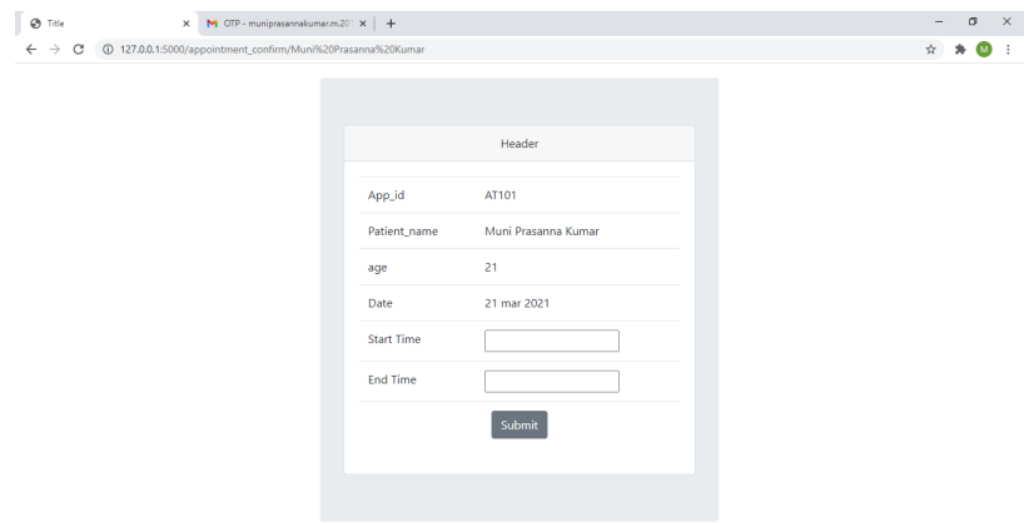

Fig 5.5 Scheduling appointments 


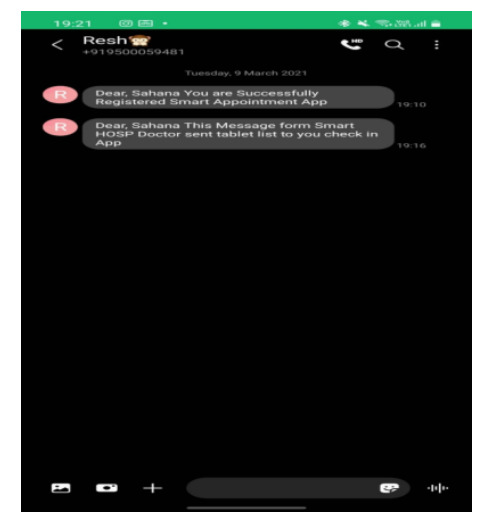

Fig 5.6 Appointment confirmation to patients

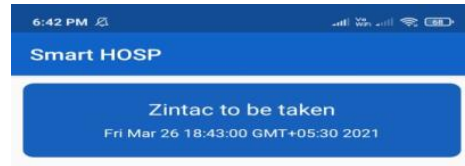

Fig 5.7 Prescription reminder 


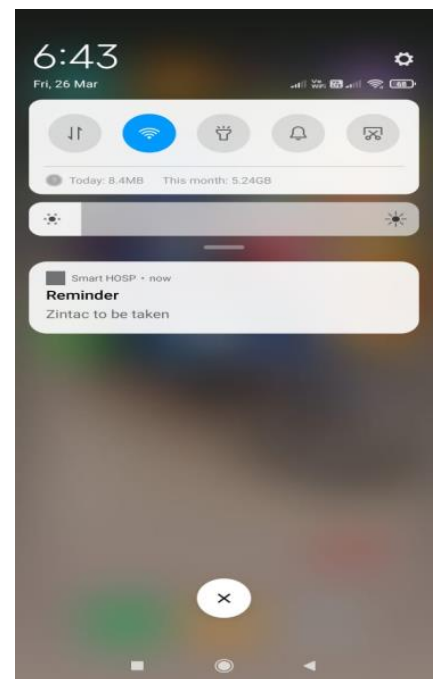

Fig 5.8 Reminder notification

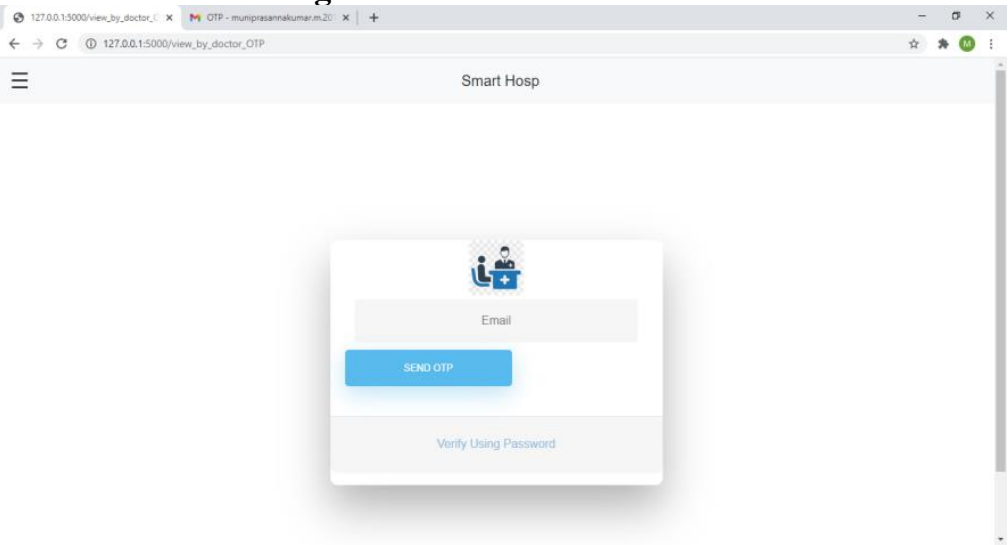

Fig 5.9 Verify using OTP
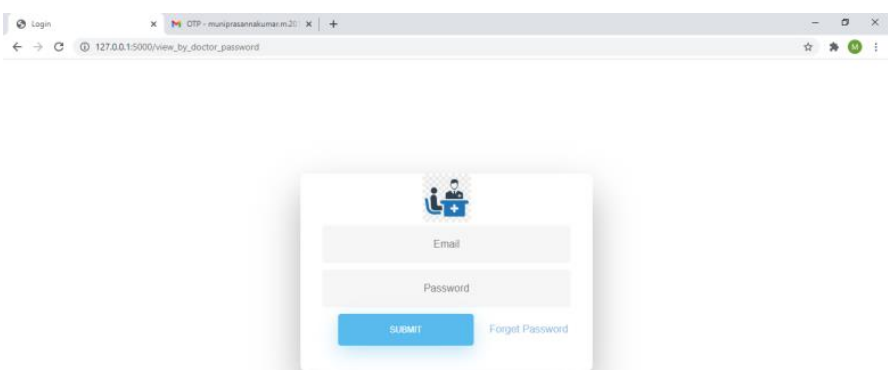

Fig 5.10 Verify using Patient credentials 


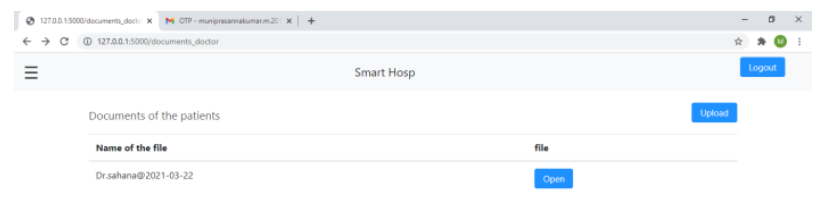

Fig 5.11 Documents of the patient

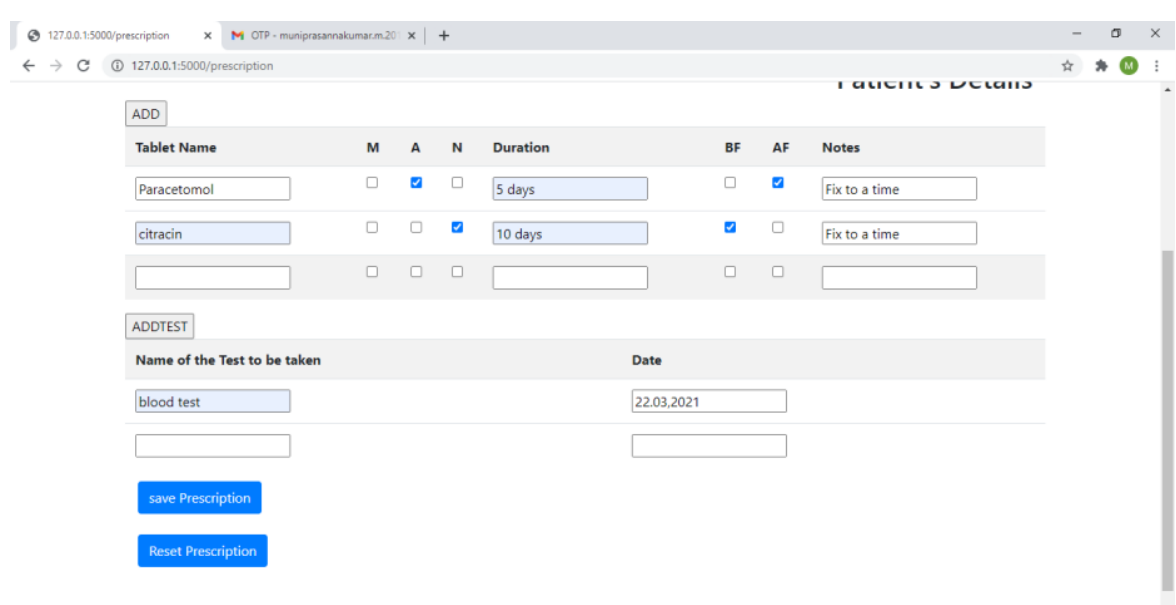

Fig 5.12 Prescription form

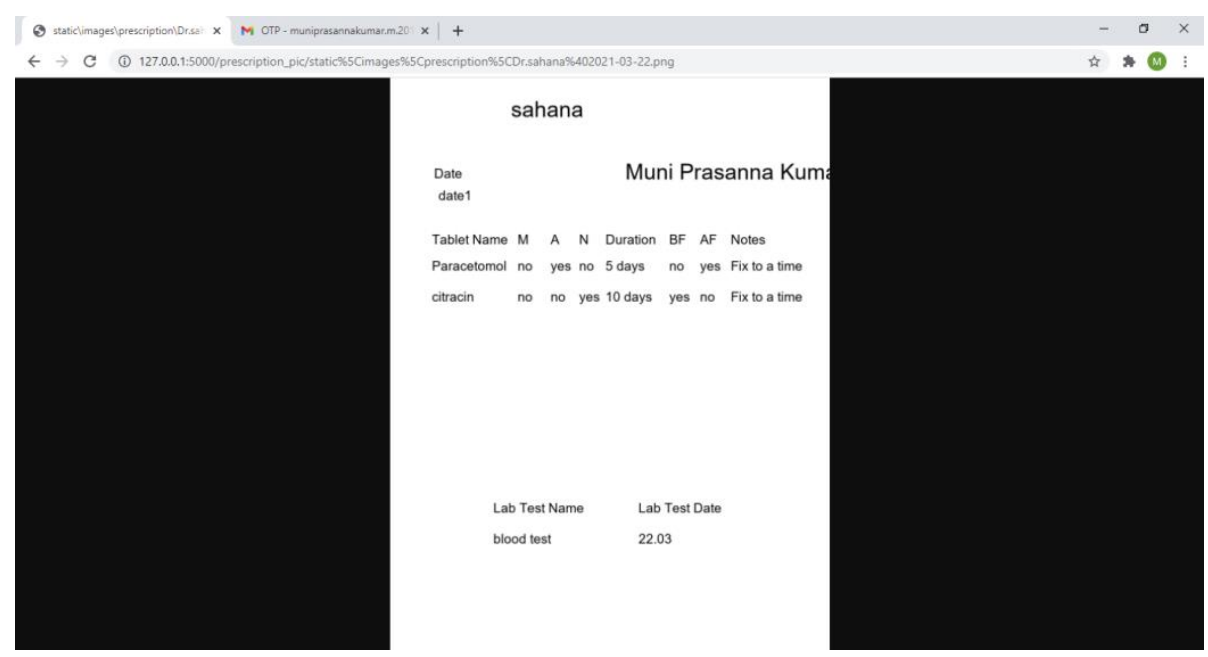

Fig 5.13 Viewing patient prescriptions 


\section{Conclusion And Future Enhancement}

\section{CONCLUSION:}

The major goal of this application is to create an interactive medium to solve the basic needs of the people. Through this app, patients can book their appointments with the doctor and they are provided with the facility to schedule alerts for all the health related reminders. It is made sure that no medical records can be tampered or accessed by intruders. To make sure that the application is available to all and easily accessible, both web and android applications are available in order for user convenience.

\section{FUTURE ENHANCEMENT:}

The system can be enhanced further in future by providing some more functionalities such as video call consultation, 24/7 chat facilities with doctor and ordering medicines through online pharmacy. More features, such as sentiment analysis for the user reviews can be added. Also, a FYI section can be added to keep the users updated about the current trends in the medical world.

\section{References}

[1] M. Amru, AllamVenkata Naga Mahesh2, PotharajuRamesh, "IoT-based Health Monitoring System with Medicine Reminder using Raspberry Pi", International Conference on Recent Advancements in Engineering and Management (ICRAEM-2020), 9-10 October 2020.

[2] V, Dr.Sangeetha and H V, Prathap and Koushik, Shreyas S and S C, Nithin and M N, Nikhil, A Smartphone Based Application for Health Catr Management Svs Tem, Institute of Scholars (InSc), August 7, 2020.

[3] MoustafaFayad, Ahmed Mostefaoui, Samir Chouali and SalimaBenbernou, "Fall Detection Application for the Elderly in the Family Heroes System", Proceedings of the 17th ACM International Symposium on Mobility Management and Wireless Access, Pages 17-23, November 2019.

[4] S.Hema Kumar, J.Uday Kiran, V.D.Ambeth Kumar, G.Saranya, Ramalakshmi V, "Effective Online Medical Appointment System", International Journal of Scientific \& Technology Research, Vol. 9, Issue 9, September 2019.

[5] Rahul Raj Verma , Harish Ramesh Varma , Mahesh ShridharKanojiya , AkshataLaddha, "Android Application of Hospital for Karjat Taluka", International Journal of Science \& Engineering Development Research, Vol.4, Issue 5, page no.120 - 122, May-2019.

[6] TabSafe :https://www.tabsafe.com/

[7] Shah MdMonirul Islam, FariaHoque Khan, Md. NajmusSwaqeeb, "An Android and Web based Application 'Urban Healthcare Service”, at BRAC University, Bangladesh, 16 April 2018.

[8] UndeGorakh Shankar, Ranjeet Kumar, Khan Abdullah Z and Asst.ProfDagadkhair Pooja, "Scan Med: A Healthcare Application using QR Code", International Journal for Research Trends and Innovation, Vol. 6, Issue 2 April 2018.

[9] Md. Abdul Majid, Mohammad Jahangir Alam and Md. Nurul Mustafa, "Smart Doctors Appointment and Prescription System", IOSR Journal of Computer Engineering (IOSR-JCE), Vol. 19, Issue 6, Nov.- Dec. 2017.

[10] Noorsyahira Ismail, ShahreenKasim, Yusmadi Yah Jusoh, Rohayanti Hassan and AyuAlyani, "Medical Appointment Application", Acta Electronica Malaysia (AEM), November 2017.

[11] MedMinder :https://www.medminder.com/

[12] Pillboxie :https://apps.apple.com/us/app/pillboxie/id417367089 
[13] Ahmed Imteaj and Muhammad Kamrul Hossain, "A Smartphone based Application to Improve the Health Care System of Bangladesh", at International Conference on Medical Engineering, Health Informatics and Technology (MediTec), 17-18 December 2016.

[14] MayureshWaykole, Vatsalya Prakash, Himanshu Singh, Nalini N, "ArduMed - Smart Medicine Reminder for Old people", International Journal of Scientific \& Engineering Research, Vol. 7, Issue 5, May-2016.

[15] IrinSherly. S ,Mahalakshmi. A ,Menaka. D, Sujatha. R," Online Appointment Reservation and Scheduling for Healthcare- A Detailed Study", International Journal of Innovative Research in Computer and Communication Engineering, Vol. 4, Issue 2, February 2016.

[16] DeeptiAmeta, KalpanaMudaliar and Palak Patel, "Medication Reminder and Healthcare - An Android Application", in International Journal of Managing Public Sector Information and Communication Technologies, Vol. 6, No. 2, June 2015.

[17] Muhammad WasimMunir, Syed Muhammad Omair, M. ZeeshanUlHaque, "An Android based Application for Determine a Specialized Hospital Nearest to Patient's Location”, International Journal of Computer Applications, Vol. 118 - No. 9, May 2015.

[18] iTriageHealth :http://myhealthapps.net/app/details/65/itriage

[19] ArvieCarpio, Jessub Kim, RashinaHoda, Duncan Miller, "MedTouch: Towards the Development of Smartphone-based Software Solutions for Mobile Health Care", in Proceedings of Australasian Software Engineering Conference, 4-7 June 2013.

[20] MediSafe :https://www.medisafe.com/

[21] Article: https://www.healthcareglobal.com/technology-and-ai-3/most-healthcare-apps-haveweak-security-report-finds 